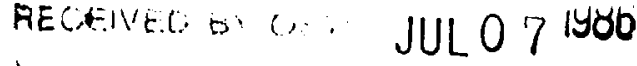 \\ Mictel \\ TITLE A SIMPLE DEFENSE CONSERVATION MOEDL FOR MASS REQUIREMENTS OF \\ HYPERVELOCITY PROJECTILE IMPACT SHIELDS FOR REENTRY VEHICLES
}

LA-UR--86-2112

AUTHOR(S) G. R. Sp111man, DRA/NDR

DE86 012407

\author{
SUEMITtED to 1986 Hypervelocity Impact Symp., October 21-24, 1986, \\ San Antonio, TX \\ Journal of Impact. Englneering, Volume 5
}

\title{
DISCLAIMER
}

\begin{abstract}
This report was prepared as an uccount of work sponsored by an agency of the United Siates Government. Neither the United State: Government nor any agency thereof, nor any of their employees, makes any warranty, express or implied, or assumes any legal liability or responsibility for the accuracy, completeness, or usefulness of any information, apparatus, product, $n$ process disclosed, or represents that its use would not infringe privately owned rights. Reference herein to any specific commarcial product, process, or service by trade name, trademark, manufacturer, or otherwise does not necessarily constitute or imply its endorsement, recom. mendation, or favoring by the United States Guvernment or any agency thereor. The views and opinions of authors expreased herein do not necessarily state or reflect thowe of the $U$. itod States Government of any agency theresf.
\end{abstract}

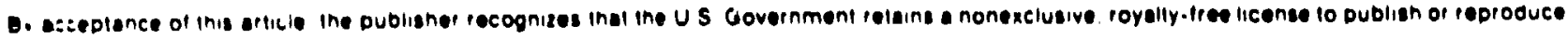

in sublished torm of inis contribution of to aliow others to do 20 . lor US Government purposes

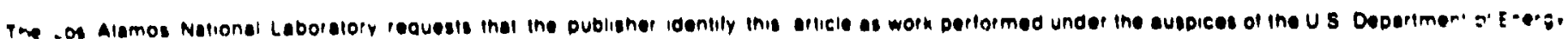




\section{A SIMPLE DEFENSE CONSERVATIVE MODEL FOR MASS REQUIREMENTS OF HYPERVELOCITY PROJECTILE IMPACT SHIELDS FOR REENTRY VEHICLES}

George R. Spll1man

Los Alamos National Laboratory, Los Alamos, NM 87544

ABSTRACT

Simple analytical modeling of the physics of interaction of hypervelocity $(50-100 \mathrm{~km} / \mathrm{s})$ projectiles with a bumper shield countermeasure is given. The interaction of projectile and bumper expansion between bumper and underlying vehicle and interaction of bumper/projectile debris cloud with vehicle are examined. Projectile/bumper interactions are treatad with ideal gas strongshock and rarefaction equations. Projectile shock decay from bumper rarefaction is approximated by an impulsive shock similarity solution. A crude model for edge rarefactions is derived. Expansion of debris is treated as an expansion superimuosed upon a translation with partition derived from a simple inelastic collision model. The effect of nonunity aspect ratlo of compressed debris is ircluded. Debris colliding elastically with the vehicle will impart momentum equal to twice the incident normal component. Impulse may be reduced up to a factor of 2 by stagnation radiative losses for small projectiles and large bumper/vehicle stand-off. Impulse can be Inhanced by vehicle ablation from radiative coupling, shock heating (inadequate stand-off), or liquid droplet microcratering (Inadequate bumper thickness). Estimates of required bumper mass are given for a spectelc example. 
George R. Spillman, 1986 HVIS, Abstract 5706 (GRS:86-08), p. 2

\section{INTRODUCTION}

We consider the physics of the problem of bumper shield countermeasuring reentry vehicles aǵainst high hypervelocity $(>50 \mathrm{~km} / \mathrm{s})$ defensive projecti.es. The hypervelocity impact bumper is a concept borrowed from tine design of meteoroid impact protection for spacecraft (Zukas and colleagues, 1982). The concept as applied to a conical reentry vehicle (RV) is illustrated in Fig. 1, in which we indicate a small, high-velocity projectile incident upon a tnin balloon (the bumper) surrounding an impulse-hardened conical RV. The strong shock interaction between bumper and projectile causes them to vaporize, which results in an expanding vapor cloud that blast-loads the underlying vehicle. The basic assumption of the projectile interaction with the vehicle is changed from that of a solid, penetiating collision to that of the blast load from a low-density cloud, which makes the interaction problem more analogor:s to that of the impulse loading because of $x$ rays.

In the following we will consider the example of tungsten for both incident projectile and bunper shield. We will assume a simple right circular cylinder with unit aspect ratio (length - ciameter) incident normally with its circular face strlking the bumper. Since the speciflc kinetlc energy of the projectile is well above vaporization energy, we will use ideal gas equations for treating the varlous interactions with nominally $\gamma-1.5$. We w11. use a rough estimate (Bennett, May 1984) of 19 Mbar as the minimum shock pressure for which tungsten completely vaporizes on relief. 
George R. Spillman, 1986 HVIS, Abstract 5706 (GRS:86-08), P. 3

\section{INTERACTION OF BUMPER AND PROJECTILE}

The impact produces equal strong shocks in the bumper and projectile with equal thermal and translational velocities, and with a compression of about a factor of 5 for $\boldsymbol{\gamma}-1.5$ is appropriate for tungsten at these high velocities). Conditions behind the shock will be modified by edge rarefactions and, after the shock emerges from bumper or back of the projectile, it will be modified by rarefactions from those surfaces. We first consider the effects of rarefaction from the back of the bumper, ignoring effects of edge rarefactions.

The various velocities of concern are $v_{p}-$ projectile velocity, $u_{p}=v_{p} / 2=$ directed velocity behind the shock - thermal rms velocity behind the shock, $v_{s}=$ shock velocity $-[(\gamma+1) / 2] u_{m}-[(\gamma+1) / 4] v_{p}$, and $C_{A}=\operatorname{adiabatic}$ speed of sound behind the shock. Here we cunsider the length of the projectile to be $\ell$ and the bumper thickness to be $\in \ell$.

We now consider the minimum bumper thickness such that a rarefaction from the back surface of the bumper cannot catch up with the shock in the profectile. The times for the shock to reach the back of the profectile and bumper, respectively, are

$$
t_{p}^{s}=\ell / v_{s}
$$

and

$$
t_{B}^{s}-\epsilon l / v_{s}
$$


George R. Spillman, 1986 HVIS, Abstract 5706 (GRS:86-08), p. 4

where $v_{s}$ is the shock speed. Behind the shock the compression and speed of sound, respectively, are

$$
\eta=\frac{\eta+1}{\eta-1}
$$

and

$$
C_{A}=\sqrt{\frac{x(y-1)}{2}}\left(\frac{x+1}{2}\right)
$$

Then the total time for the shock to reach the back of the bumper and the following rarefaction to reach the rear of the projectile is

$$
\mathrm{t}_{\mathrm{R}}^{\rho}-\frac{\epsilon \ell}{\mathrm{v}}+\frac{1+\epsilon) \ell}{\eta \mathrm{C}_{\mathrm{A}}}-\frac{\ell}{\mathrm{v}_{\mathrm{s}}}\left(\epsilon+(1+\epsilon) \overline{\left.\sqrt{\frac{\gamma-1}{2 \gamma}}\right]}\right.
$$

The time to penetrate the distance $\epsilon \ell$ is

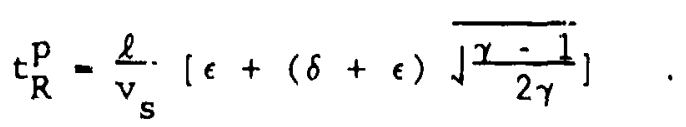

The rarefaction catches the shock when $\delta \ell-V_{S} t_{R}^{p}$, or when

$$
\delta=\frac{1+\sqrt{(\gamma-1) / 2 \gamma}}{1-\sqrt{(\gamma-1) / 2 \gamma}},(-2.38 \text { for } \gamma-1.5)
$$


George R. Spillman, 1986 HVIS, Abstract 5706 (GRS:86-08), p. 5

We will now assume that the similarity solution for an impulsively loaded, free surface, holds for the shock once the bumper rarafaction has overtaken the shock; that is,

$$
P_{S}-X^{-n}
$$

where $\mathrm{n}$ is a weak function of $\gamma$, having values of $4 / 3,1.275$, and 1.191 at $\gamma$ $=1.4,5 / 3$, and 2.8 , respectively. The use of

$$
P_{S}=P_{S}^{0}\left[\frac{(\delta+\epsilon) \ell}{(1+\varepsilon) \ell}\right]^{n}-P_{S}^{0}\left[\frac{3,4 \epsilon}{1+\epsilon}\right]^{4 / 3}
$$

In the absence of a rarefaction from the back of the bumper or projectile, the head of the edge rarefaction from the contac surface intersects the shock at a radius

$$
r-r_{p}-v_{s} t \overline{\sqrt{\frac{\gamma-1}{\gamma+1}}}
$$

which has the value $r-0.1 r_{p}$ when the shock reaches the rear of our nominal unit \&spect ratio projectile. Therefore, edge rarefacions are important-even for our nominal projectile. We now attempt a crude quantiflcation of the effect of the erge rarefaction on shock decay in the projectlle.

Recall the equations for the ldiabatic rarefaction of an ideal gas bnunded Initially at $x-0$ are

$$
\frac{c}{c_{0}}-\frac{2}{\gamma+1}-\frac{\gamma-1}{\gamma+1} \frac{x}{C_{0} t}-\text { adiabatic speed of sound }
$$


George R. SpIllman, 1986 HVIS, Abstract 5706 (GRS:86-08), p. 6

$$
\begin{array}{ll}
\rho=\rho_{0}\left(\frac{C}{C_{0}}\right)^{2 /(\gamma-1)} & =\text { density } \\
F=P_{0}\left(\frac{C}{C_{0}}\right)^{2 \gamma /(\gamma-1)} & - \text { pressure } \\
I=I_{0}\left(\frac{C}{C_{0}}\right) & =\text { Internal energy },
\end{array}
$$

where the subscript zero refers to the undisturbed region. For a planar rarefaction, we obtain the average pressure between $x=-C_{0} t$ (head of the rarefaction) and $X-0$ (edge of projectile) as

$$
\bar{P}-\frac{P_{0}}{C_{0} t} \int_{-C_{0} t}^{0}\left[\frac{2}{\gamma+1}-\frac{\gamma-1}{\gamma+1} \frac{x^{2}}{C_{0} t}\right]{ }^{2 \gamma /(\gamma-1)} d x
$$

We now assume that this planar rarefaction holds behind our shock front in a cylindrical geometry and that the reduced pressure is immediately communtcated to the shock front. If the rarefaction has traveled the distance dx $+C_{0} d t$, the average pressure over the whole shock front is

$$
\overline{\mathrm{p}}-\overline{\mathrm{p}}\left[1-\left(1-\frac{\mathrm{dx}}{r_{\mathrm{p}}}\right)^{2}\right]+\mathrm{p}_{0}\left(1-\frac{\mathrm{dx}}{r_{p}}\right)^{2} .
$$

We obtaln as estimates of the effect of tige rarefaction on the average shock pressure

$$
P-P_{0} e^{-0.43} \int^{v_{s} d t / r_{P}} \quad(\gamma-1.5)
$$


George R. Spillman, 1986 HVIS, Abstract 5706 (GRS:86-08), p. 7

Recently, The edge effect on shock decay was investigated (Taylor, October 1985) using steady-state $2 \mathrm{D}$ approximation and obtained a different form--but similar quantitative results for the snock decay. We assume for our nominal projectile $\left(l-2 r_{p}\right) c k .=$ the net effective decay is given by the product of planar and edge effect decay. If we require tinat the rear surface shock be greater than vaporization pressure, :re have

$$
\mathrm{P}_{\mathrm{S}}^{0}\left(\frac{3.4 \epsilon}{1+\epsilon}\right)^{4 / 3} \geq 2.36 \mathrm{P}_{\mathrm{V}}
$$

or

$$
\epsilon \geq\left[3.4\left(\frac{\mathrm{P}_{S}^{0}}{2.26 \mathrm{P}_{\mathrm{v}}}\right)^{-1}-1\right]
$$

When the above condition is not satisfied, we may expect a portion of tha projectile to emerge as liquid or solid rather than vapor, which has implications for interactions with the underlying structure.

We will make a "cookie cutter" approximation to determine the mass of bumper pitncipally involved in the collision; 1.e., we assume that the bumper mass involved in the collision is that in direct line of the incoming projectile. A mass, $m_{p}$, is incilent and a mass, $m_{p}(1+\epsilon)$ emerges containing the incident momentum and energy. Note that this assumption pertains only to the mass sharing the main energy and monentum and not to the size of a hole produced in the bliner. One-dimensional synthesis suggests that at our high velocities, less than 2.58 of the energy is transferred laterally into a 
George R. Spillman, 1986 HVIS, Abstract 5706 (GRS:86-08), p. 8

bumper of $\epsilon-1 / 4$ and 2D steady-state approximations (Taylor, June 1985) and 2D hydrodynamics calculations (Oyer, 1985) support the cookie-cutter assumption.

VUID REGION EXPANSION

As the projectile and ejected bumper material emerge from the back of the bumper, it is compressed and heated, but still has a net translational velocity. Some of the existing heat energy will be converted to kinetic energy of expansion and the overall expansion may be viewed as a superposition of the net translational velocity and the local expansion velocity.

We consider first the unreal case of a spherical expansion in which the material emerges as a sphere with half the energy in translational and half in internal energy with all internal convertible to expansion kinetic energy. If the sphere expands as a thin shell, then the shell remains tangent to the bumper at $\sigma=i 30^{\circ}$ (see Fig. 2) and expands at the projectile velocity $\left(v_{p}\right)$ at $\theta-0^{\circ}$. In the center-of-mass frame, the expanding balloon has a uniform mass distribution in solid angle. The emerging debris will not be in the form of a sphere. If we could ignore the edge rarefactions until the shocks emerged from front and back, for the case of $\epsilon-1$ the emerging debris could be characterized as a disk of diameter equal to the projectile diameter and a length 408 of the projectile length (a linear compression of twice the projectile length by a factor of 5 ). We could then expect translational and expansion velocities to each be one-half of the profectile velocity, but the uniformity of mass distribution with solid angle would be lost. Since rarefactions in any direction will move the same 
George R. Spiliman, 1986 HVIS, Abstract 5706 (GRS:86-08), P. 9

distance In a given amount of time, the mass moving in a particular direction will be approximately proportional to the area of the compressed debris normal to that direction. For a thin face, however, the effective area wi1l be reduced about a factor of 2 by rarefactions from other face. From this discussion, we make en assumption that the radio of $\mathrm{dm} / \mathrm{d} \cos \theta$ in the axial and radial directioons is proportional to areas normal to $\theta-0^{\circ}$ and $\theta=90^{\circ}$ gives

$$
\left(\frac{\mathrm{s} m}{\mathrm{~d} \cos \theta}\right)_{0^{\circ}} /\left(\frac{\mathrm{dm}}{\mathrm{d} \cos \theta}\right)_{90^{\circ}}-\mathrm{r}_{\mathrm{p}} / \bar{l} \text { where } \bar{l} \text { - disk thickness. (20) }
$$

We define $\mu-\cos \theta$ and arbitrarily fit the two directions with the form $a+$ b $\mu$. Additionally, we assume that the expansion is as from a disk starting at uniform compression $\eta$, given from the strong shock equations such that $\bar{l}-\ell_{p}(1+\epsilon) / \eta$, and using $\eta-5$ for $\gamma=1.5$. We obtain

$$
\frac{d m}{d \mu} \sim 1+\mu^{2}\left(\frac{1,5-\epsilon}{1+\epsilon}\right)
$$

We now consider a very simple inelastic collision model for the effect of bumper thickness on expansion velocity. We assume that the expansion will again be uniform in direction in the center-of-mass coordinate system. Defining $v_{P}^{\prime}$ as center-of mass translacional veloctty and $v_{T}$ as expansion velocity allows us to obtain from energy and momentum conservation

$$
v_{p}^{\prime}=v_{p} /(1+\epsilon) ; v_{T}-v_{p} e^{1 / 2} /(1+\epsilon)
$$

and simple geometry gives (see Fig. 2) 
George R. SpI1lman, 1986 HVIS, Abstract 5706 (GRS:86-08), p. 10

$$
(\tan \phi)_{\max }=\epsilon^{1 / 2} \sin \theta /\left(1+\epsilon^{1 / 2} \cos \theta\right)
$$

and the maximum value is

$$
(\tan \phi)_{\max }-\sqrt{\epsilon /(1+\epsilon)} \text { at } \theta-\cos ^{-1}(-\sqrt{\epsilon})
$$

The Item of most interest in the expansion is the angular distribution of the axial component of the momentum. This we may write as

$$
\frac{d\left(m v_{N}\right)}{d \cos \phi}-v_{N} \frac{d m}{d \mu} / d(\cos \phi) / d \mu
$$

where $v_{N}$ is the axial velocity given by

$$
v_{N}-v_{N}^{\prime}+v_{T} \cos \theta-v_{P}\left(1+\epsilon^{1 / 2} \cos \theta\right) /(1+c)
$$

under assumption of expansion as a thin shell at velocity $v_{T}$ in all directions from the center of mass. Since $\theta$ is louble valued for any $\phi$, there are two values to sum for Eq. (25). From Eq. (23), we obtain

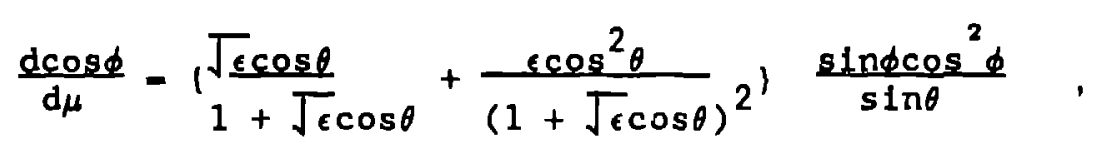

which is most convenierit to use in this mixed form. We use Eqs. (21), (23), (25), (26), and (27) to numerically evaluate $d m v_{N} / d \cos \theta$ and normalize to the incident momentum; this is plotted in F1g. 3. The plots show a peak at $\phi-$ $\phi_{\max }$ because of the assumption of infinitesimal debris shell thlckness. 
George R. Spillman, 1986 HVIS, Abstract .5706 (GRS:86-08), P. 11

Finite thickness will tend to remove this double-peaked structure. We may note the tendency toward uniform distribution for $\phi<\phi_{\max }$ for $\operatorname{smal1} \epsilon$, even for the infinitesimal shell thickness. Note also that the strict limitaticn of debris to angles $\phi \leq \phi_{\max }$ is an artifact of assigning the rms value of velocity to expansion for all mass points. From Eq. (24), we may obtain for $\operatorname{smal1} \epsilon$

$$
\begin{aligned}
\frac{1}{m_{p} v_{p}} \frac{d m v_{N}}{d \cos \theta} & =\frac{2}{\epsilon} & \phi<\phi_{\max }(\epsilon<<1) \\
& =0 & \phi>\phi_{\max }
\end{aligned}
$$

INTERACTION OF DEBRIS WITH UNDERLYING STRUCTURE

The debris cloud striking the underlying structure may interact elastically or inelastically. If the collision is elastic, twice the normal component of the incident momentum will be delivered to the structure. The momentum transfer to the structure may be reduced to the normal incident component if the debris can radiate away all internal energy on stagnation without caus ing any ablation of the underlying structure. The momentum may be enhanced by severa! possible mechanisms causing ablation, including shock heating, radiative transfer from the stagnating debris, and microcratering by liquid droplets or solid fragments. We assume that avoidance of each of these potential momentum enhancement mechanisms is a countermeasure design goal. We discuss each mechanism to some degree. 
George R. Spillman, 1986 HVIS, Abstract .5706 (GRS:86-08), p. 12

To be able to estimate impact and stagnation densities and times, we will assume that the expending debris shell is 0.2 times a radius thick. Shock ablation may be circumvented by achieving a large density discontinuity between the debris cloud and the material of the underlying structure. This is achieved by debris cloud expansion via an adequate com ination of sefaration between bumper and underiying stiucture and bumper thickness. Our calculations indicate that reducing the incident vapor impulse/area to a reasonable level seems likely to avoid siock ablation. At least, it is defense conservative to assume so.

At $70 \mathrm{~km} / \mathrm{s}$ impact velocity, the stagnation temperature of a low- 2 debris cloud may be of order $3 \times 10^{5}$, and that of a high-z debris cloud may be twice as high. At such high temperatures, there is a possibility of significant radiative energy transfer. The maximum radiative flux from a stagnating layer is the blackbody value. For a layer of optical thickness $\tau \ll 1$, the flux is reduced approximately by the factor $2 \tau$. For $T \gg 1$, the radiative rate is reduced by the diffusion process. For a slab ir steady-state radiating from one face with a uniform heat source, the radiati'e flux is given by

$$
F=\frac{20 T^{4}}{1+3 / 4 T}
$$

where $\sigma$ is the Stefan-Boltzmann constant and $T$ is the internal temperature at optical depth $T$. [This is derivable arlalagously to the Milne protiem diffusion solution (Zel'dovich and Raizer, 1966) by setting the divergence of the flux equal to a nonzero constant.) 
George R. SpiJ.1man, 1986 HVIS, Abstract 5706 (GRS:86-08), p. 13

We have used Eq. (29) with strong shock equations, tcgether with a factor for radiative collapse to give stagnation density, temperature, and radiative flux--and somewhat arbitrarily defined the condition of radiative flux at une-half stagnation time becoming larger than onc-ha!f of rate of kinetic energy flux into the stagnation region as a transition from nonradiative stagnation to radiative stagnation. Using this procedure, we find that those expansions to Low density (and large area) tend to lose energy radiatively; those of lesser expansion do not, e.g., far tungsten projectiles in the mass range of 1 to $10 \mathrm{~g}$, stagnations on underlying structure such that the momentum/area incident is less than about 4 ktap tend to lose energy radiatively. On structures closer such that incident momentum is much higher, radiative loss is small.

For high-density particles incident (such as liquid droplets) ejecta from craters can give significant momentum enhancement (e.g., about a factor of 5 for profectiles incident on aluminum ac $70 \mathrm{~km} / \mathrm{s}$ ) (Dienes and Walst, 1970). The minimum size of an atomic cluster that acts as a cratering projectile may be estimaied by finding the minimum aggregate of atoms that have suffi. cient energy melt a hamisphere of depth equal to the atomic range. Such estimates of minimum mass coirezponds to large molecules.

A more restrictive condition on the size of liquid droplets that may give momentum enhancement comes from a requirement that they must be abie to penetrate the stagnating vepor. This condition we may express in terms o: the vapor impulse/area and projectile velocity. The areal density of stag. nating vapor is $\mathrm{m} / \mathrm{A}-\mathrm{L} / \mathrm{v}_{\mathrm{p}}$, where $\mathrm{L}$ is the moinertum/area in the stagnating vapor. The droplets w111 penetrate the stagnating vapor effectively if 
George R. Spil1man, 1.986 HVIS, Abstract 5706 (GRS:86-08), F. 14

$$
\rho_{D} R_{D} \gg \frac{1}{2} m / A=L / 2 v_{p}
$$

where $\rho_{D}$ and $R_{D}$ are droplet density and radius, respectively; i.e., if $R_{D} \gg$ $L / 2 \rho v_{p}$ or, equivalently, $m_{D} \gg \pi L^{3} / 6 \rho^{2} v_{p}^{3}$. For $v_{p}-70 \mathrm{~km} / \mathrm{s}, \rho-$ $19.3 \mathrm{~g} / \mathrm{cm}^{2}$ and $\mathrm{L}=10^{5}$ taps, this requirement is $\mathrm{m}_{\mathrm{D}} \gg 4 \times 10^{-12} \mathrm{~kg}$. Unless the droplets are vaporized in the stagnation layer before reaching the underlying structure, even very smali droplets can cause momentum enhancement by microcratering. The effect of possible droplec vaporization by the radiation field or other heat transfer in the stagnation layer has not been adequately cunsidered and may lead to requirements of larger droplet masses for microcratering. For the present, we assume that the bumper is failing to perform adequarely if the shock in the projectile drops below that required for full vaporization on release.

\section{APPLICATIONS}

We have applied the above physics to estfinating minimum bumper mass to reduce blast loading below an assurned lethal impulse/area level for projectiles of varlous mass and velocities incident on a specifir target. The target selected is a cone of $2 \mathrm{~m}$ length and $10^{\circ}$ cone half-angle. The mass of the bumper shield w11l be its areal density times tes area. For very small stand-off $(h)$ the area essentially does not vary with increasing $h$, but tho impulse/area falls off as $h^{-2}$. In such a region, the bumper wolght can be decreased by reducing thickness and increasing stand-off (provlded that we do not drop below the full vaporlantlo" regime). At large standoff, the aren Increases an $h$ ? and, usling, our nsymptottc expression for the angular detrlbution of momentum [Bq. (2R)], we seo there 1 s no further galn 
George R. Sp111man, 1986 HVIS, Abstract. 5706 (GRS:86-08), p. 15

to be made by increasing the stanbd-off further. But, also, we limit the bumper thickness according to Eq. (19). For projectile masses of $1 \mathrm{~B}$ or more or impulse levels below $120 \mathrm{ktap}$, this limit does not have a large effect on bumper mass estimates.

This minimization process has been applied to estimation of required bumper shell mass 3 optimized against specific projectile liass and velocity for the conical vehicle described above. Some results for a profectile velocity of $50 \mathrm{~km} / \mathrm{s}$ and an impulse loading of $120 \mathrm{ktap}$ on the underlying vehicle are shown in Fig. 4. Here, the curve labeled leakage probability of 1 - corresponds to normal impact of the projectile as modeled herein. Other values of leakage probability correspond to the probability of the angle of incidence for a random direction of the profectile, resulting in i.ess than 120-ktap impulse. In modeling oblique impact, we assume that tha effective thickness of the bumper is increased by the reciprocal of the cosine of the angle between the trafectory and the normal to the surface and that the angular distribution about the trajectory line is unchanged. Since the debris cloud (KInslow, 1970) is deflected toward the normal, we believe this results in an overly conservative estimate of leakage. 
George R. Spillman, 1986 HVIS, Abstract. 5706 (GRS:86-08), p. 16

\section{REFERENCES}

Zukas, J. A., Nicholas, T., Swift, H. F., Greszczuk, L. B., and Curran, D.R. (1982). Impact Dynamics, John Wiley and Sons, New York.

Swift, H. F., Bamford, R., and Chen, R. (March 1, 1982). Designing dual. plate meteoroid shields--A new analysis, JPL Publication 82-39,

Bennett, B., (May 1984). Personal communication, Los Alamos National Laboratory.

Taylor, J. W. (October 4, 1985). Alternatives (without prejudice) to Splilman's Bumper Mechanics, Los Alamos National Laboratory inemorandum ADDRP/JWT: $85-39$.

Taylnr, J. W. (June 1986). Personal communication, Los Alamos National Laboratory.

Oyer, A. T. (May 1986), Personal communication, Los Alamos National Laboratory.

Zel'dovich, Y. B. and Raizer, Y. P. (1966). Physics of shock waves and h1gh-temperature hydrodynamic phenomena, Vol. I, Academic Press, New York.

Dienes, J. K. and Wal.sh, J. M. (19,0). In R. Kinslow (Ed.) High-Velocity Impact Phenomena, A.cademic Press, New York.

Gehring, J. W. Jr. (1.970). In R. Kinslow (Ed.) High-Vejoctty Impact Phenomena, Academic: Press, New York. 


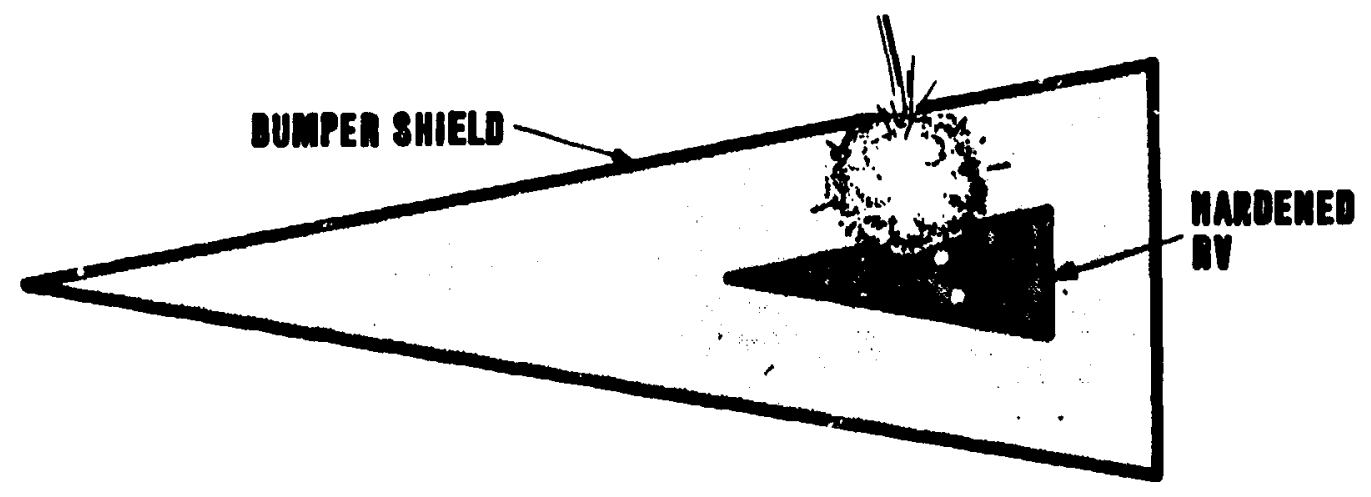

Fig. 1. The bunper shleld concept.

\section{THE DEBRIS EXPANSION}
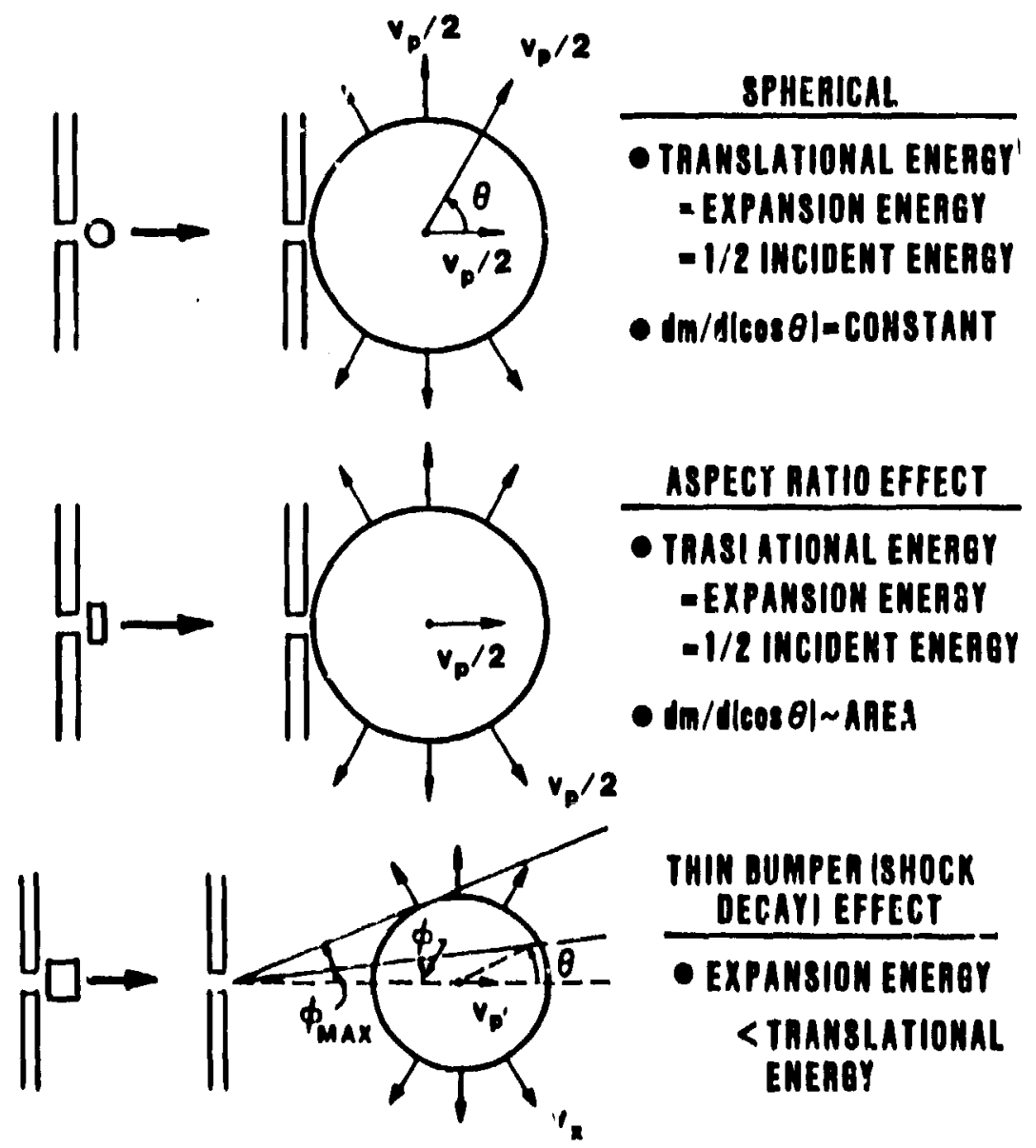

F1g. 2. The debris expansion. 


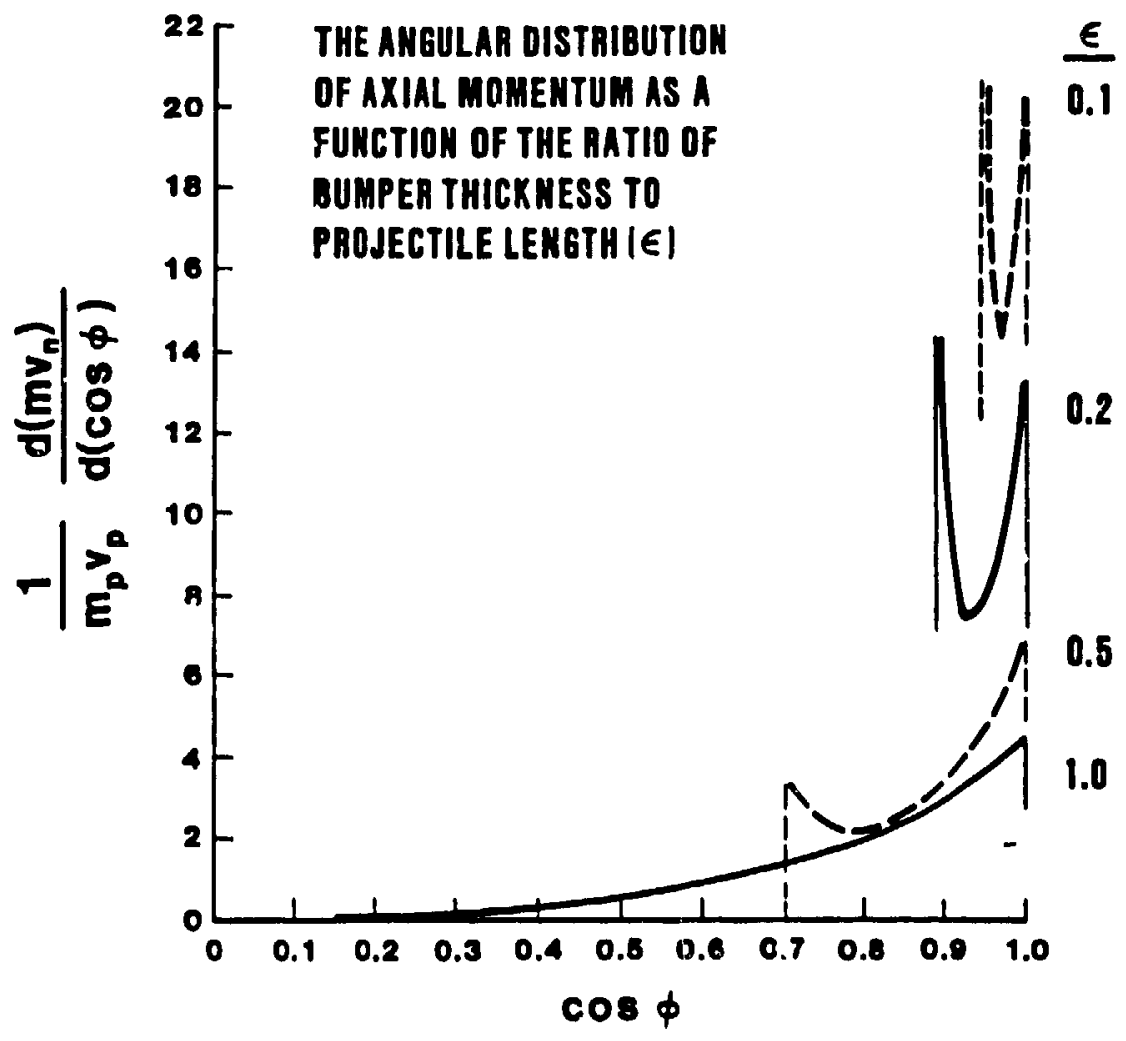

Fig. 3. The angular distribution of axial momentum as a function of the ratio of bumper thickness to projectile length (c).

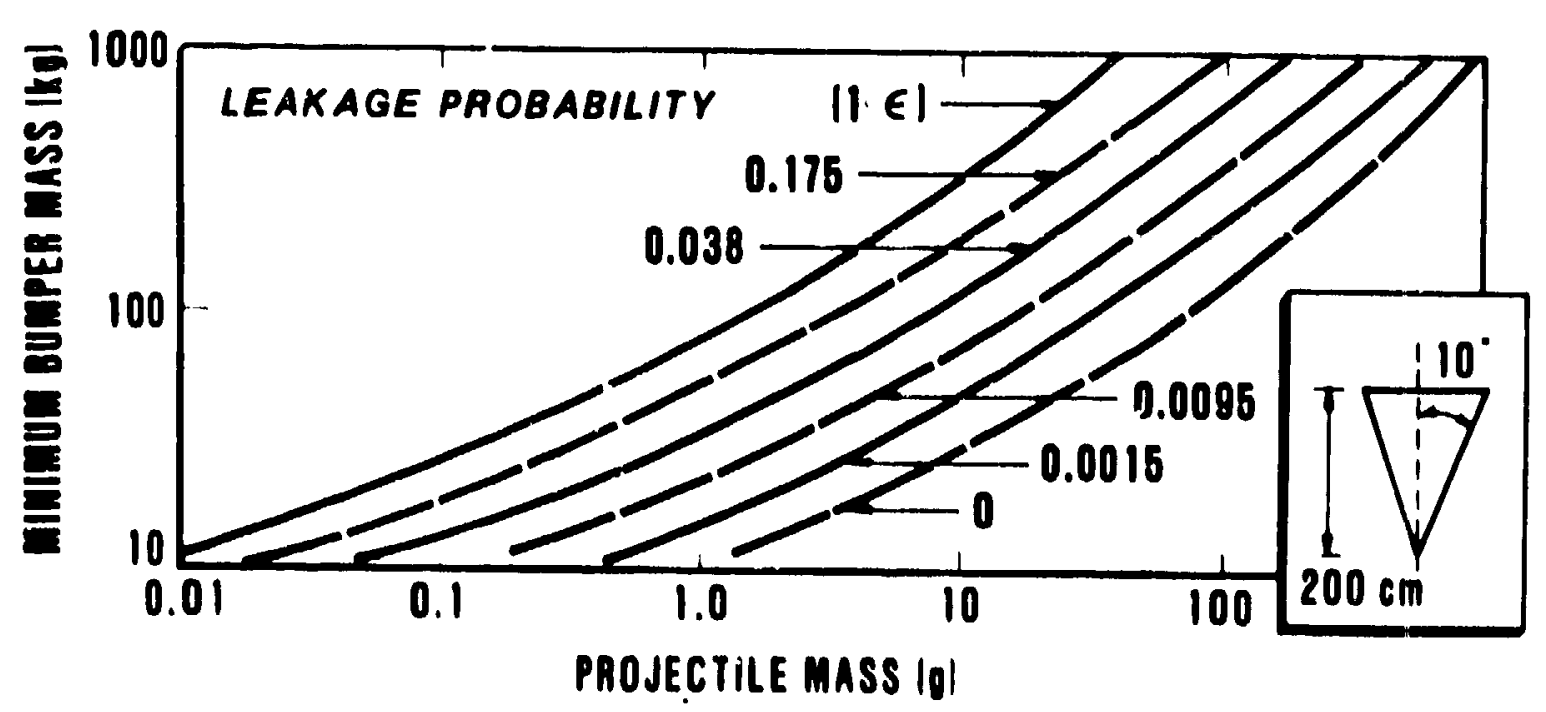

F18. 4. Minimum required bumper mass to achleve speclfled survival (leaknge) probabllity agalnat random-direction hit of tungaten projectile with velocity of $50 \mathrm{~km} / \mathrm{s}$. 